INDO GLOBAL JOURNAL OF

PHARMACEUTICAL SCIENCES

ISSN 2249- 1023

\title{
Molecular and Proteomics Analysis of Glucosyltransferases from Gymnema sylvestre R.Br. Playing a Pivotal Role in Sterol Metabolism
}

\author{
Pragya Tiwari ${ }^{*}$, Neelam S. Sangwan \\ Department of Metabolic and Structural Biology, CSIR-Central Institute of Medicinal and Aromatic Plants (CIMAP), P.O.- CIMAP, \\ Lucknow-226015 (U.P.), India
}

Address for Correspondance: Pragya Tiwari, pragyamita02@gmail.com

\begin{abstract}
Keywords Diabetes mellitus;

Glucosyltransferase

; Gymnema

sylvestre R.Br.;

Triterpene

Glycoside; Sterol

Glycosides

Biosynthesis.
\end{abstract}

\begin{abstract}
Sterol glycosyltransferases are important class of enzymes, catalyzes glucosylation of triterpenoids and steryl glucosides formation in planta. The addition of a carbohydrate moiety to secondary metabolites enhances several biological properties as food additives and as key drugs or drug-derivatives in pharmacological studies. Gymnema sylvestre R.Br. a pharmacologically important antidiabetic herb, attributed to the presence of bioactive triterpene glycosides. Although some information regarding pharmacology and phytochemical profiles of the plant is available, no attempts have been made so far to decipher the biosynthetic pathway and key enzymes involved in biosynthesis of steryl glucosides. The presentation discusses the identification and catalytic characterization of a glucosyltransferase, catalyzing biosynthesis of steryl glycosides. The full length cDNA (2572 bp) contained an open reading frame of 2106 nucleotides, was heterologously expressed in bacterial expression system and the recombinant protein encoded a polypeptide of 77 kiloDalton as observed on SDS-PAGE. The GsSGT was expressed in Escherichia coli and biochemical characterization of the recombinant enzyme suggested its key role in the biosynthesis of steryl glucosides with catalytic preference for C-3 hydroxyl group of sterols. This SGT from G. sylvestre R.Br. is unique as it possesses the capacity to glucosylate different kinds of sterols present in lower organisms like bacteria and fungi to higher organisms (plants and animals). This study highlights significant possibilities focusing on sterol metabolism and its evolution from microbes to higher organisms and would further aim at pathway engineering of sterol metabolism. (C) 2016 iGlobal Research and Publishing Foundation. All rights reserved.
\end{abstract}

Conference Proceedings: International Conference on Advances in Plant and Microbial Biotechnology (PMB2017); JIIT, Noida: February 02-04, 2017

Indo Global Journal of Pharmaceutical Sciences( ISSN 22491023 ; CODEN- IGJPAI; NLM ID: 101610675) indexed and abstracted in EMBASE(Elsevier), SCIRUS(Elsevier),CABI, CAB Abstracts, Chemical Abstract Services(CAS), American Chemical Society(ACS), Index Copernicus, EBSCO, DOAJ, Google Scholar and many more. For further details, visit http://iglobaljournal.com 